Nota

\title{
ESPECTROSCOPIA DE INFRAVERMELHO NA DETERMINAÇÃO DA TEXTURA DO SOLO ${ }^{(1)}$
}

\author{
Tatiana Maris Ferraresi (2), Wilson Tadeu Lopes da Silva ${ }^{(3)}$, Ladislau Martin-Neto(4), Pedro \\ Marques da Silveira ${ }^{(5)} \&$ Beáta E möke Madari ${ }^{(6)}$
}

\begin{abstract}
RESUMO
A aplicação de técnicas espectroscópicas que utilizam a radiação infravermelha (NIRS-Near Infrared Spectroscopy e DRIFTS-Diffuse Reflectance Fourier Transformed Spectroscopy) na análise inorgânica do solo tem sido proposta desdea década de 1970, mas até os dias atuais são raros os métodos implementados rotineiramente no Brasil. Isso deve-se à dificuldade em construir modelos de calibração, por meio de métodos estatísticos multivariados, utilizando-se amostras reais de solo, de constituição complexa, que varia geograficamente e de acordo com o manejo. Por isso, os objetivos destetrabalho foram construir modelos de cal ibração em NI RS e DR IFTS para a quantificação das frações de argila e areia, em amostras de solos de classes diferentes - Latossolo Vermelho (predominante), Nitossolo, Argissolo Vermelho e Neossolo Quartzarênico - eavaliar qual dessas duas técnicasé mais adequada para essa finalidade, assi $m$ como a interferência do agrupamento de amostras e da seleção de variáveis espectrais na qualidade desses modelos. Para isso, valores de referência obtidos pelo método do densímetro, método largamente utilizado nos laboratórios de análise de solo, foram correlaci onados com valores de absorbância em NIRS e DRIFTS pela ferramenta estatística PLS (Partial Least Squares), obtendo-se altos coeficientes de determi nação $\left(R^{2}\right)$, de 0,95, 0,90 e 0,91 para argila, silte e areia, respectivamente, na validação externa. Isso confirma a aplicabilidade das técnicas espectroscópicas na análise granulométrica do solo para fins agrícolas. $O$ agrupamento das amostras segundo a localização e a seleção de variáveis espectrais pouco influenciou na qualidade dos modelos. A técnica espectroscópica mais indicada para essa finalidade foi a DRIFTS.
\end{abstract}

Termos de indexação: granulometria do solo, técnicas espectroscópicas, radiação infravermelha, Latossolo, argila.

\footnotetext{
(1) Recebido para publicação em 05 de dezembro de 2011 e aprovado em 20 de setembro de 2012.

(2) Mestre em Química Analítica, Embrapa Arroz e Feijão, Rodovia GO 462, km 12. CEP 75375-000 Santo Antônio de Goiás (GO). E-mail: tatiana.ferraresi@embrapa.br

(3) Doutor em Química Analítica, Embrapa Instrumentação, Rua XV de Novembro 1452. CEP 13560-970 São Carlos, (SP). E-mail: wilson.Iopes-silva@embrapa.br

(4) Doutor em Física Aplicada, Embrapa Instrumentação. E-mail: Iadislau.martin@embrapa.br

(5) Doutor em Fertilidade de Solos e Nutrição de Plantas, Embrapa Arroz e Feijão. E-mail: pedro.silveira@embrapa.br

(6) Doutora em Ciência do Solo e Nutrição de Plantas, Embrapa Arroz e Feijão. E-mail: beata.madari@embrapa.br
} 


\section{SUMMARY: INFRARED SPECTROSCOPY IN DETERMINATION OF SOIL TEXTURE}

Sincethe 70s, spectroscopic techniques, especially thosewith infrared radiation (NI RS Near Infrared Spectroscopy and DRIFTS - Diffuse Reflectance F ourier Transformed Spectroscopy) have been proposed for inorganic soil analysis, but only few methods found routineusein Brazil so far. This fact is probably dueto thedifficulty in building cali bration models based on multivariate tools with real soil samples that havea compl ex composition that varies geographical ly and according to themanagement. Theobjective of this study was to build modes for day, silt and sand fractions of soils from two experimental farms of Embrapa, and assess theinfluenceof theclusters of samples and spectral variablesel ection on themode quality. For this purpose, referencevalues of day, silt and sand werecorrelated with NIRS and DRIFTS absorbancevalues by the statistical tool of Partial least-squares (PLS) modeling. High regression coefficients were obtained $(0.95,0.90$ and 0.91 for clay, silt and sand, respectively), in theexternal val idation. Theseresults confirm theapplication of spectroscopic techniques on textural analysis of soil for agricultural purposes. Thegrouping of samples by location and thesel ection of spectral variables had littleinfluenceon themodel quality. The most suitablespectroscopic techni quefor this purposewas DRI FTS.

Index terms: soil texture, spectroscopy techniques, infrared radiation, Latosol, clay.

\section{NTRODUÇÃO}

A textura é uma característica importante no manejo dos sol os, pois determina, em grande parte, o grau de coesão e adesão entre as partículas do solo. Os teores relativos das partículas do sol o influenciam na taxa de infiltração eretenção deágua, na aeração ena disponibilidade de nutrientes (F orsythe, 1975). Além disso, a sua determinação no sol o permitefazer uso mais racional e eficiente dos fertilizantes (Santos et al., 2010).

Os métodos normalmente utilizados na determinação dos teores de argila, silte e areia baseiam-se na velocidade de sedimentação das partículas que compõem o sol o quando suspensas em água após dispersão quími ca efísica (Embrapa, 1997). A quantificação das partículas dispersas érealizada retirando-sealíquota com pipeta, seguida de secagem epesagem dosolo, ou simplesmentepor meio da medida da densidade. Esses métodos envolvem um preparo inicial de amostras para a dispersão, podendo ser realizadas em torno de 30 amostras por dia.

De acordo com Farmer \& Palmieri (1975), a espectroscopia no infravermel ho é uma das poucas técnicas que podem render informação sobre a estrutura e a composição de fases amorfas, como o solo moído, sendo uma ferramenta útil na caracterizaçãomolecular deespécies inorgânicas. Mais de uma centena de amostras podem ser analisadas por dia, com a possibilidade de alimentar um amostrador automático acoplado ao equi pamento.

Os espectros de infravermel ho são resultantes de diferentes tipos de vibração molecular. Enquanto o espectro noinfravermelho médio com refletância difusa apresenta os sinais referentes às vibrações fundamentais das moléculas, no infravermelho próximo detectam-se as vibrações harmônicas e as combinações.

Essas técnicas vibradionais, queutilizam a radiação infravermel ha para excitar as moléculas, são comuns em muitos laboratórios etêm certas vantagens sobre alguns métodos tradicionais de análise, como, por exemplo: a pequena quantidadedeamostra requerida, a rapidez, a facilidade de preparo deamostra eo curto tempo de análise. O número deamostras analisadas podeser triplicado, com redução decusto, pois el imina ouso de reagentes dispersantes. Ela também permite oestudosimultâneo deespécies orgânicas einorgânicas (Reig et al., 2002), sendo bem atrativa para o monitoramento ambiental, modelagem eagricultura de precisão (Viscarra-Rossel et al., 2006).

Muitos estudos têm demonstrado que a espectroscopia derefletância di fusa no infravermel ho fornece sinais marcantes, que podem ser usados para predizer muitos atributos físicos, químicos ebiológi cos do solo (Madari et al., 2006; Viscarra-Rossel et al., 2006; Minasny et al., 2008). Entre os atributos físicos já estudados estão a superfície específica eas frações de areia, siltee argila, sendo a maioria dos trabalhos realizada no sentido de determinar a granulometria do solo (Malley et al., 2004; Reeves III, 2010). Essa determi nação podeser mais precisa em sol os cujoteor de matéria orgânica nãoultrapassa $5 \%$.

Diversos fatores relacionados à formação dos solos são determinantes para a feição espectral: rocha de origem (que pode levar a uma diferença no tipo de argila formada, segundo Resende et al., 2005), topografia, material vegetal eclima. Por esse motivo, Vuuren et al. (2006) sugerem utilizar amostras de um mesmo campo experimental nas cali ibrações por Espectroscopia de I nfravermelho Próximo (NIRS) ou Espectroscopia de I nfravermel ho Médio (DRIFTS), 
para construir bons modelos preditivos ao correlacionar os dados de absorbância com os dados físicos dosolo.

Em razão da variedade de matrizes de solo, decorrentedos diferentes processos deformaçãodestes, podehaver problemas com ouso dessa técnica (Malley et al., 2004). A heterogeneidade das amostras, aliada à característica indireta de determinação por infravermelho, implica a necessidade de grande número de amostras de cal ibração e de separação das amostras por localidade (Vuuren et al., 2006). Uma alternativa para superar o problema de matriz é sel ecionar regiões do espectro mais específicas para as propriedades quesedeseja quantificar.

A melhor calibração é aquela que alia o maior coeficiente de determinação ao menor SEP (erro de predição), com o menor número defatores necessários. Os obj etivos destetrabal ho for am construir model os decalibraçãoem NIRS eDRIFTS para a quantificação das frações de argila, silte e areia, em solos de diferentes classes, com predominância de Latossolo Vermelho, eavaliar a interferência do agrupamento de amostras e da sel eção de variáveis espectrais na qualidade desses modelos.

\section{MATERIAL E MÉTODOS}

F oram col etadas amostras superficiais, $0-10 \mathrm{~cm}$ de profundidade, de solos de áreas experimentais pertencentes à E mbrapa Arroz eF eijão (CNPAF) eà Embrapa Pecuária Sudeste (CPPSE), Iocalizadas, respectivamente, em Santo Antônio de Goiás - GO (16

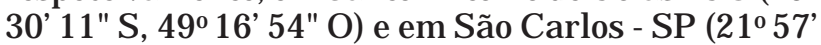
$\left.35^{\prime \prime} \mathrm{S}, 47^{\circ} 50^{\prime} 32^{\prime \prime} \mathrm{O}\right)$.

F oram obtidas amostras do solo sob diferentes utilizações naquel emomento: solos sob cultivo dearroz, feijão, café, banana e pastagens, além de solos sob vegetação nativa de cerrado, nas áreas da Embrapa Arroz e Feijão (amostras CNPAF ). J á na Embrapa Pecuária Sudeste (CPPSE) foram amostrados sol os sob cultivo decana-de-açúcar, alfafa, aveia e pastagens acrescentadas de amostras de matas com vegetação nativa de Cerrado e Mata Atlântica (amostras CPPSE). O total de amostras analisadas foi de 120 (70 amostras do CNPAF e 50 amostras do CPPSE ).

As áreas experimentais do CNPAF são constituídas, em sua maior parte, de Latossolo Vermelho, mas amostras de solo foram coletadas também em $\mathrm{N}$ itossol o. As col etas no CPPSE foram no Latossolo Vermelho (predominante), Argissolo Vermelhoe NeossoloQuartzarênico.

Para fins de calibração, as amostras foram agrupadas de acordo com a locali dade em CNPAF e CPPSE. Os cálculos estatísticos para obtenção de model os de cal ibração foram realizados com estes dois grupos separadamente, assim como com todas as amostras (TODOS).
O método do densímetro foi utilizado para determinar a granulometria do solo, conforme E mbrapa (1997). A densidade da suspensão de solo (1.000 mL) foi determinada, após agitação manual, em dois tempos: 30 s (D1) e 2 h (D2), e a fração de cada classe de partícula foi cal culada pel as equações 1 a 3:

$$
\begin{gathered}
\text { Argila }\left(\mathrm{g} \mathrm{kg}^{-1}\right)=\left[\left(D_{2} \times 100\right) / 50\right] \times 10 \\
\text { Areia }\left(\mathrm{g} \mathrm{kg}^{-1}\right)=\left\{100-\left[D_{1} \times 100\right] / 50\right\} \times 10 \\
\text { Silte } \left.\left(\mathrm{g} \mathrm{kg}^{-1}\right)=\left\{\left(D_{1}-D_{2}\right) \times 100\right] / 50\right\} \times 10
\end{gathered}
$$

Para obtenção dos espectros de solos, as amostras foram secas ao ar, trituradas em moinho de bolas e passadas em peneiras de $0,250 \mathrm{~mm}$. A proximadamente $1 \mathrm{~g}$ de cada amostra foi colocado em um frasco de vidro de fundo chato e analisado em espectrômetro no infravermel ho próximo (10.000 a $4.000 \mathrm{~cm}^{-1}$ ) Perkin Elmer Spectrum 100, de forma que a radiação infravermelha inci disse no fundo dos frascos de vidros, atingindo as amostras superficialmente. Para anál ise em espectrômetro de infravermel ho médio (4.000 a $400 \mathrm{~cm}^{-1}$ ) Varian 600IR, aproximadamente $0,20 \mathrm{~g}$ de amostras de sol o foi acondicionado em recipientes e colocado em um amostrador automático (Pike) de forma que olaser incidisse na superfície das amostras e a radiação refletida fosse captada por um espelho côncavo. A resolução na obtenção dos espectros foi de $16 \mathrm{~cm}^{-1}$, acumulando-se 32 varreduras por espectro. F oram obtidos espectros de refletância transformados para absorbância pela equação $A=\log (1 / R)$. Todos os espectros for am centrados na média esubmetidos ao cál culo da primeira derivada com cinco pontos.

O método PLS com validação cruzada (leave-one out) foi aplicado em 80 \% das amostras de cada agrupamento (CNPAF e CPPSE) para construir model os de cal i bração; os $20 \%$ restantes tiveram seus valores preditos pelos model os construídos (vali idação externa). Essas amostras de vali idação externa foram escolhidas a partir de gráficos de distribuição, provenientes de uma análise de componentes principais (PCA) com amostras de cada subgrupo, selecionando-se $20 \%$ das amostras de forma representativa (Figura 1 ). Em toda a análise estatística utilizou-se o software Pirouette versão 3.11 .

Com oagrupamento detodas as amostras, também foram testados três subgrupos devariáveis espectrais (números deonda) na faixa do infravermelho próximo (NIRS) e na faixa do infravermel ho médio (DRIFTS). Os critérios de seleção de variáveis espectrais em DRIFTS eNIRS são apresentados no quadro 1.

Os parâmetros utilizados para comparar a eficiência de cada modelo de calibração construído foram: fatores necessários na cali ibração (NF); número de amostras (NvceNve); desvios-padrão de predição na validação cruzada e externa (SEV e SEP); erros percentuais de predição(SEV\% ESEP\%); ecoeficientes de determinação linear entre os dados preditos e 


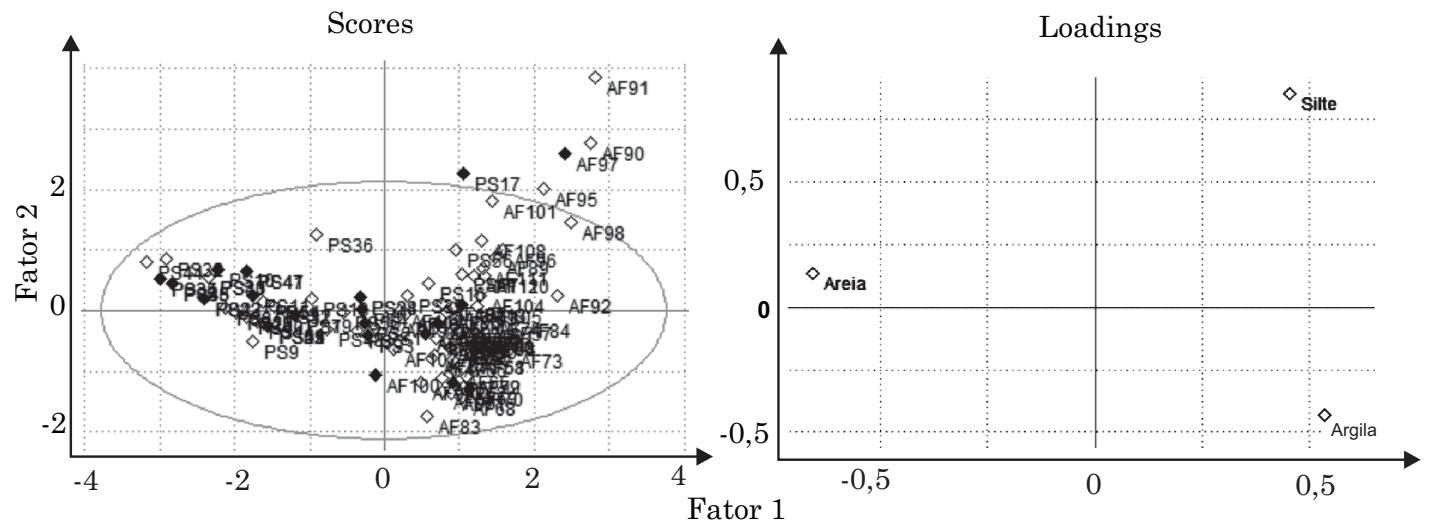

Figura 1. Gráficos de distribuição das amostras (scores) com base nas quantidades de areia, silte e argila (loadings) provenientes da análise de componentes principais (PCA). Í cones preenchidos representam amostras selecionadas para a validação externa.

\section{Quadro 1. Critérios de seleção de variáveis espectrais}

\begin{tabular}{|c|c|c|}
\hline DRIFTS & Região do espectro retirada & Critério \\
\hline Seleção 1 & 2113,5 a $3293,8 \mathrm{~cm}^{-1}$ & Retirar sinais provenientes de matéria orgânica. \\
\hline Seleção 2 & 1712,4 a $3347,8 \mathrm{~cm}^{-1}$ & Retirar interferentes orgânicos, inclusive sinais de carbonilas. \\
\hline Seleção 3 & 1203,3 a $1697 \mathrm{~cm}^{-1}$ e 2013,3 a $3694,9 \mathrm{~cm}^{-1}$ & $\begin{array}{l}\text { Utilizar apenas sinais de argilominerais e quartzo, retirando } \\
\text { inclusive bandas referentes a aminas, amidas e hidroxilas. }\end{array}$ \\
\hline \multicolumn{3}{|l|}{ NIRS } \\
\hline Seleção 1 & 10000 a $7202 \mathrm{~cm}^{-1}$ e 6998 a $4702 \mathrm{~cm}^{-1}$ & $\begin{array}{l}\text { Retirar regiões sem bandas e aquelas atribuídas a moléculas de } \\
\text { água livre. }\end{array}$ \\
\hline Seleção 2 & 10000 a $7202 \mathrm{~cm}^{-1}$ e 6648 a $5350 \mathrm{~cm}^{-1}$ & $\begin{array}{l}\text { Retirar apenas regiões sem bandas (ruídos e diferenças no } \\
\text { espalhamento da luz). }\end{array}$ \\
\hline Seleção 3 & 10000 a $4704 \mathrm{~cm}^{-1}$ & $\begin{array}{l}\text { Utilizar somente região de predominância de argilominerais, } \\
\text { como caulinita e gibbsita. }\end{array}$ \\
\hline
\end{tabular}

DRIFTS = espectroscopia de infravermelho médio; NIRS = espectroscopia de infravermelho próximo.

medidos (Rvc e Rve). Os erros de predição foram calculados a partir das seguintes equações:

$$
\begin{array}{cc}
\operatorname{SEP}=\sqrt{\frac{1}{\mathrm{Nvc}}} \sum_{\mathrm{i}=1}^{\mathrm{n}}(\mathrm{y}-\hat{\mathrm{y}})^{2} \quad \mathrm{SEV}=\sqrt{\frac{1}{\mathrm{Nve}}} \sum_{\mathrm{i}=1}^{\mathrm{n}}(\mathrm{y}-\hat{\mathrm{y}})^{2} \\
\operatorname{SEP}(\%)=\frac{\operatorname{SEP}}{\overline{\mathrm{y}}} \times 100 \quad \operatorname{SEV}(\%)=\frac{\operatorname{SEV}}{\overline{\mathrm{y}}} \times 100
\end{array}
$$

em que Nvc = número de amostras utilizadas na validação cruzada; Nve = número de amostras utilizadas na validação externa; $y=$ val or medido com método de referência, $\bar{y}=$ val or médio para $\hat{y}$ dentro do grupo amostral utilizado na calibração; $\hat{y}=$ valor predito pel o model o de cal ibração.

Após a determinação dos teores de argila, siltee areia pel o método de referência (densímetro) e pela espectroscopia, as amostras reservadas para a validação cruzada foram classificadas quanto à textura, utilizando-se o diagrama triangular das classes texturais do solo (Prevedello, 1996).

\section{RESULTADOSE DISCUSSÃO}

Os resultados da análise granulométrica são apresentados no quadro 2. Observa-se que os sol os do CNPAF são, em média, mais argilosos, enquanto as amostras doCPPSE contêmmai or valor médio deareia.

SegundoSousaJ únior et al. (2008), os constituintes mais importantes e característicos queinfluenciam a intensidadeda refletância ea feição espectral sãoa areia, osilte, minerais como quartzo, magnetita, caulinita e esmectita, a matéria orgânica e o ferro total, que permitem a caracterização ea discriminação dos solos.

$\mathrm{Na}$ figura 2, observam-se, nos espectros obti dos por DRIFTS, sinais referentes a ligações normalmente presentes em compostos que constituem a matéria orgânica do solo, principal menteem torno de $2.900 \mathrm{~cm}^{-1}(v \mathrm{C}-\mathrm{H}), 3.150 \mathrm{~cm}^{-1}(v \mathrm{O}-\mathrm{H}$ evN-H), 1.630 $\mathrm{cm}^{-1}(v \mathrm{C}=\mathrm{C}$ de aromáticos e $v \mathrm{C}=\mathrm{O}$ de amida I e carboxilatos) e $1.450 \mathrm{~cm}^{-1}(\delta \mathrm{N}-\mathrm{H}$ de amida II) (Gerzabek et al., 2006). 
Quadro 2. Valor mínimo, valor máximo e média de concentração dos elementos texturais nos solos das duas áreas experimentais

\begin{tabular}{lcrcr}
\hline \multirow{2}{*}{ Solo } & \multirow{2}{*}{ Elemento textural } & \multicolumn{3}{c}{ Valor } \\
\cline { 3 - 5 } & & Mínimo & Máximo & Média \\
\hline \multirow{3}{*}{ CNPAF kg-1 $^{-1}$} & 296 & 679 & 580 \\
& Argila & 7 & 374 & 106 \\
\multirow{2}{*}{ CPPSE } & Silte & 167 & 657 & 316 \\
& Areia & 96 & 486 & 309 \\
& Argila & 7 & 237 & 85 \\
& Silte & 357 & 867 & 634 \\
\hline
\end{tabular}

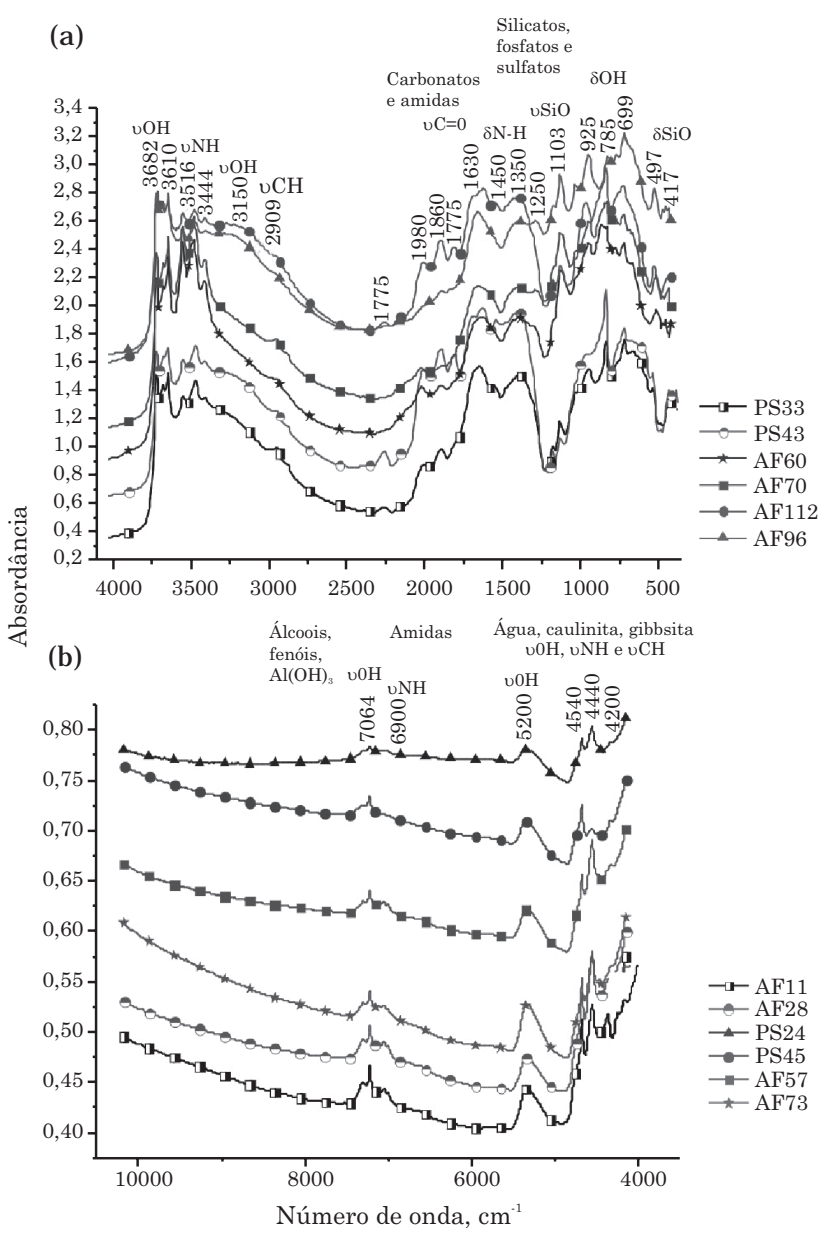

Figura 2. Exemplos de espectros de absorbância em infravermelho médio - DRIFTS (a) e infravermel ho próximo - NIRS (b) de solos do CNPAF (representados por AF) e do CPPSE (representados por PS).

De acordo com Farmer \& Palmieri (1975), as principais vibrações de filossilicatos podem ser separadas em vibrações de estiramento (v) evibrações angulares ( $\delta$ ) de hidroxilas e óxido de silício: $v \mathrm{OH}$
(3.400-3.750 $\left.\mathrm{cm}^{-1}\right), 8 \mathrm{OH}\left(600-950 \mathrm{~cm}^{-1}\right)$, vSi-O $(700-$ $\left.1.200 \mathrm{~cm}^{-1}\right)$ e $\delta$ Si-O $\left(150-600 \mathrm{~cm}^{-1}\right)$. J á as bandas em $1.050 \mathrm{~cm}^{-1}$ e entre 900 e $400 \mathrm{~cm}^{-1}$ são atribuídas a minerais deargila e ao quartzo (Pirie et al., 2005).

Observa-se(Figura 2a) queabsorções de carbonatos próximas a $1.400 \mathrm{~cm}^{-1}$ são separadas de ânions silicatos, fosfatos esulfatos que aparecem sobrepostos na região de 1.200 a $900 \mathrm{~cm}^{-1}$. Há indícios de que o espectronoinfravermelhoé particularmentesensível à desor dem na estrutura cristal ina, conforme ocorre diminuição da formação cristalina ou substituição isomórfica (F armer \& Palmieri, 1975).

Nos espectros deNIRS (F igura 2b) são observadas principal mente as vibrações de estiramento (v) das ligações $\mathrm{O}-\mathrm{H}, \mathrm{N}-\mathrm{H}$ eC-H. As bandas em torno de 7.000 $\mathrm{cm}^{-1}$ em geral são resultantes dos estiramentos de hidroxilas, o que pode indicar a presença de fenois, ál coois e hidróxidos de alumínio; estes últimos compõem argilominerais, como caulinita e montmorilonita. Estiramentos da ligação $\mathrm{N}-\mathrm{H}$, que compõe ureia e proteínas, também são observados nessa região.

Bandas dealta absorção centradas em $5.200 \mathrm{~cm}^{-1}$ estão relacionadas com moléculas de água livre (normalmenteadsorvidas) presentes em minerais 2:1 (Demattê et al., 2004).

Na região de $4.200-4.500 \mathrm{~cm}^{-1}$ são detectados sinais devO-H provenientes decaulinita egibbsita, além de vibrações vN-H evC-H.

Os resultados da cal ibração com a ferramenta PLS aplicada aos dados espectroscópicos e aos dados de referência mostraram quetantoNIRS quantoDRIFTS predisseram satisfatoriamente os teores deareia, silte e argila (Quadro 3), sendo observados resultados ligeiramente melhores com os espectros de infravermel ho médio, assim como havia sido descrito por Viscarra-Rossel et al. (2006). Os coeficientes de determinação (Rvc e Rve) obtidos com todas as amostras, com e sem seleção de variáveis, foram superiores aos alcançados por aqueles autores em DRIFTS (argila $=0,67$, silte $=0,49$ e areia $=0,74$ ) e por Chang et al. (2001) em NIRS (argila $=0,67$, silte $=0,84$ eareia $=0,82$ ) e comparáveis aos coeficientes al cançados por Madari et al. (2006) em DRIFTS (argila $=0,96$ e areia $=0,99$ ). Conforme esperado, as cal ibrações para silteresultaram em predições menos acuradas, em razãodo método dequantificaçãoindireto desse constituinte.

Como mostra o quadro 3, não houve melhora significativa nos resultados a partir da seleção de variáveis espectrais (sel eções 1, 2 e 3). I sso pode ser explicado pela impossibilidade de distinguir totalmente os compostos orgânicos de inorgânicos nos espectros de infravermel ho, pois as mesmas ligações quími cas podem estar presentes em ambos os tipos de compostos. A seleção de bandas, nesse caso, provocou perda de informação, resultando em calibrações menos precisas. 
Quadro 3. Dados de validação cruzada e externa para os modelos de cali bração em NIRS e DRIFTS para os teores de argila, silte eareia, utilizando as seleções de variáveis espectrais e todas as amostras coletadas

\begin{tabular}{|c|c|c|c|c|c|c|c|c|c|}
\hline \multirow{2}{*}{$\begin{array}{l}\text { Faixa } \\
\text { espectral }\end{array}$} & \multirow{2}{*}{ Modelo } & \multicolumn{4}{|c|}{ Validação cruzada } & \multicolumn{4}{|c|}{ Validação externa } \\
\hline & & NF & Nvc & Rvc & SEV\% & Nve & Rve & SEP & SE P\% \\
\hline \multicolumn{10}{|c|}{ Argila } \\
\hline \multirow{4}{*}{ DRIFTS } & Sem seleção & 5 & 94 & 0,86 & 78,04 & 26 & 0,95 & 55,38 & 11,90 \\
\hline & Seleção 1 & 5 & 94 & 0,86 & 78,34 & 26 & 0,95 & 55,47 & 11,92 \\
\hline & Seleção 2 & 4 & 94 & 0,85 & 80,82 & 26 & 0,93 & 64,63 & 13,88 \\
\hline & Seleção 3 & 4 & 94 & 0,82 & 88,16 & 26 & 0,92 & 67,47 & 14,49 \\
\hline \multirow{4}{*}{ NIRS } & Sem seleção & 4 & 97 & 0,85 & 83,96 & 24 & 0,86 & 82,58 & 17,74 \\
\hline & Seleção 1 & 3 & 97 & 0,84 & 85,97 & 24 & 0,85 & 85,21 & 18,30 \\
\hline & Seleção 2 & 4 & 97 & 0,85 & 84,85 & 24 & 0,85 & 84,25 & 18,10 \\
\hline & Seleção 3 & 3 & 97 & 0,85 & 85,00 & 24 & 0,85 & 83,85 & 18,01 \\
\hline \multicolumn{10}{|c|}{ Silte } \\
\hline \multirow{4}{*}{ DRIFTS } & Sem seleção & 4 & 94 & 0,67 & 46,13 & 26 & 0,50 & 59,83 & 69,49 \\
\hline & Seleção 1 & 4 & 94 & 0,67 & 46,24 & 26 & 0,50 & 59,81 & 69,45 \\
\hline & Seleção 2 & 4 & 94 & 0,66 & 46,84 & 26 & 0,47 & 61,35 & 71,25 \\
\hline & Seleção 3 & 4 & 94 & 0,62 & 49,40 & 26 & 0,52 & 57,68 & 66,98 \\
\hline \multirow{4}{*}{ NIRS } & Sem seleção & 4 & 97 & 0,55 & 53,19 & 24 & 0,83 & 40,00 & 46,45 \\
\hline & Sel eção 1 & 5 & 97 & 0,50 & 54,94 & 24 & 0,90 & 29,26 & 33,98 \\
\hline & Seleção 2 & 4 & 97 & 0,54 & 52,95 & 24 & 0,84 & 40,42 & 46,94 \\
\hline & Seleção 3 & 3 & 97 & 0,47 & 55,61 & 24 & 0,78 & 44,40 & 51,56 \\
\hline \multicolumn{10}{|c|}{ Areia } \\
\hline \multirow{4}{*}{ DRIFTS } & Sem seleção & 5 & 94 & 0,86 & 94,23 & 26 & 0,91 & 76,66 & 17,10 \\
\hline & Seleção 1 & 5 & 94 & 0,86 & 94,07 & 26 & 0,92 & 75,58 & 16,86 \\
\hline & Seleção 2 & 5 & 94 & 0,86 & 94,23 & 26 & 0,90 & 80,10 & 17,86 \\
\hline & Seleção 3 & 5 & 94 & 0,84 & 102,08 & 26 & 0,92 & 77,43 & 17,27 \\
\hline \multirow{4}{*}{ NIRS } & Sem seleção & 4 & 97 & 0,82 & 108,11 & 24 & 0,87 & 98,75 & 22,03 \\
\hline & Seleção 1 & 3 & 97 & 0,80 & 112,48 & 24 & 0,85 & 105,61 & 23,56 \\
\hline & Sel eção 2 & 5 & 97 & 0,82 & 107,13 & 24 & 0,91 & 83,05 & 18,52 \\
\hline & Seleção 3 & 3 & 97 & 0,81 & 110,88 & 24 & 0,85 & 103,72 & 23,13 \\
\hline
\end{tabular}

DRIFTS = espectroscopia de infravermelho médio; NIRS = espectroscopia de infravermelho próximo; NF = número de fatores ou componentes principais utilizados na calibração; Nvc = número de amostras preditas na validação cruzada; Rvc = coeficiente de determinação na validação cruzada; SEV = desvio-padrão da validação cruzada; Nve = número de amostras na validação externa; Rve = coeficiente de determinação na validação externa; SEP = desvio-padrão da validação externa; SEV\% e SEP\% = erros médios percentuais de predição.

O agrupamento amostral por localidade(Quadro 4) também não resultou em mel horia dos resultados preditos, devidoà heterogenei dade das amostras eao pequeno número de amostras pertencentea cada um deles. Desse modo, reunindo-se todas as amostras (Quadro 3), obtiveram-se model os de predição mais complexos e mais robustos e coeficientes de determinação próximos de 1 (Rve Argila $=0,95$, Rve Silte $=0,83$ eRve Areia $=0,91$ ).

Os mel hores model os foram obtidos com DRIFTS, que ofereceal gumas vantagens sobre NI RS no que se refereà quantidadee definição de bandas, além deser quimicamente mais sensível aos constituintes da amostra. NIRS, como mencionado por Merry \& J anik (2001), é insensível ao quartzo, um dos maiores componentes do solo, sendo mais influenciado pelo tamanho das partículas e pelos processos físicos de reflexão da luz, decorrentes da distribuição espacial das partículas de areia e argila. Por outro lado, há maior facilidadedemanuseiodeamostras pulverizadas em NIRS, além do menor custo do equi pamento e da sua manutenção (Viscarra-Rossel et al., 2006). Somamsea essas características de NIRS a disponibilidade de equipamentos portáteis para uso em campo (Viscarra-Rossel et al., 2006) ea larga utilização dessa faixa do infravermelho na obtenção de dados por satélites (Dalmolin et al., 2005; Reeves III , 2010). Por enquanto, a espectroscopia de infravermel ho médio seria mais indicada para uso em laboratório, sendo necessário um aprimoramento tecnológico mais sofisticado para uso em campo.

A figura 3 mostra os val ores preditos por model os em DRIFTS versus os valores medidos por densitometria. São apresentados os val ores preditos 
Quadro 4. Dados de validação cruzada e externa para os modelos de calibração em DRIFTS e NIRS para o teor de argila, silte e areia, utilizando agrupamentos de amostras por localidade

\begin{tabular}{|c|c|c|c|c|c|c|c|c|c|}
\hline & \multirow{2}{*}{ Modelo } & \multicolumn{4}{|c|}{ Validação cruzada } & \multicolumn{4}{|c|}{ Validação externa } \\
\hline & & $\mathbf{N F}$ & Nvc & Rvc & SEV\% & Nve & Rve & SEP & SEP\% \\
\hline \multirow{4}{*}{ ARGILA } & CNPAF DRIFTS & 3 & 55 & 0,62 & 45,90 & 15 & 0,22 & 91,66 & 15,79 \\
\hline & CPPSE DRIFTS & 1 & 38 & 0,79 & 75,42 & 12 & 0,10 & 71,67 & 23,16 \\
\hline & CNPAF NIRS & 4 & 55 & 0,36 & 65,80 & 15 & 0,16 & 66,05 & 11,38 \\
\hline & CPPSE NIRS & 1 & 40 & 0,74 & 76,35 & 11 & 0,62 & 82,70 & 26,72 \\
\hline \multirow{4}{*}{ SILTE } & CNPAF DRIFTS & 4 & 55 & 0,69 & 52,02 & 15 & $-0,15$ & 57,79 & 55,90 \\
\hline & CPPSE DRIFTS & 2 & 38 & 0,45 & 40,32 & 12 & $-0,08$ & 31,81 & 56,30 \\
\hline & CNPAF NIRS & 2 & 55 & 0,67 & 52,91 & 15 & 0,64 & 40,92 & 39,58 \\
\hline & CPPSE NIRS & 1 & 40 & 0,35 & 42,60 & 11 & $-0,26$ & 32,92 & 58,27 \\
\hline \multirow{4}{*}{ AREIA } & CNPAF DRIFTS & 8 & 55 & 0,58 & 46,50 & 15 & 0,31 & 105,68 & 33,41 \\
\hline & CPPSE DRIFTS & 2 & 38 & 0,80 & 93,02 & 12 & 0,13 & 75,40 & 11,89 \\
\hline & CNPAF NIRS & 3 & 55 & 0,47 & 64,86 & 15 & 0,19 & 68,30 & 21,59 \\
\hline & CPPSE NIRS & 1 & 40 & 0,69 & 104,14 & 11 & 0,53 & 97,59 & 15,39 \\
\hline
\end{tabular}

CNPAF = Centro Nacional de Pesquisa em Arroz e Feijão; CPPSE = Centro Nacional de Pesquisa em Pecuária Sudeste; DRIFTS = espectroscopia de infravermelho médio; NIRS = espectroscopia de infravermelho próximo; NF = número de fatores ou componentes principais utilizados na calibração; Nvc = número de amostras preditas na validação cruzada; Rvc = coeficiente de determinação na validação cruzada; SEV = desvio-padrão da validação cruzada; Nve = número de amostras na validação externa; Rve = coeficiente de determinação na validação externa; SEP = desvio-padrão da validação externa; SE V\% e SEP\% = erros médios percentuais de predição.
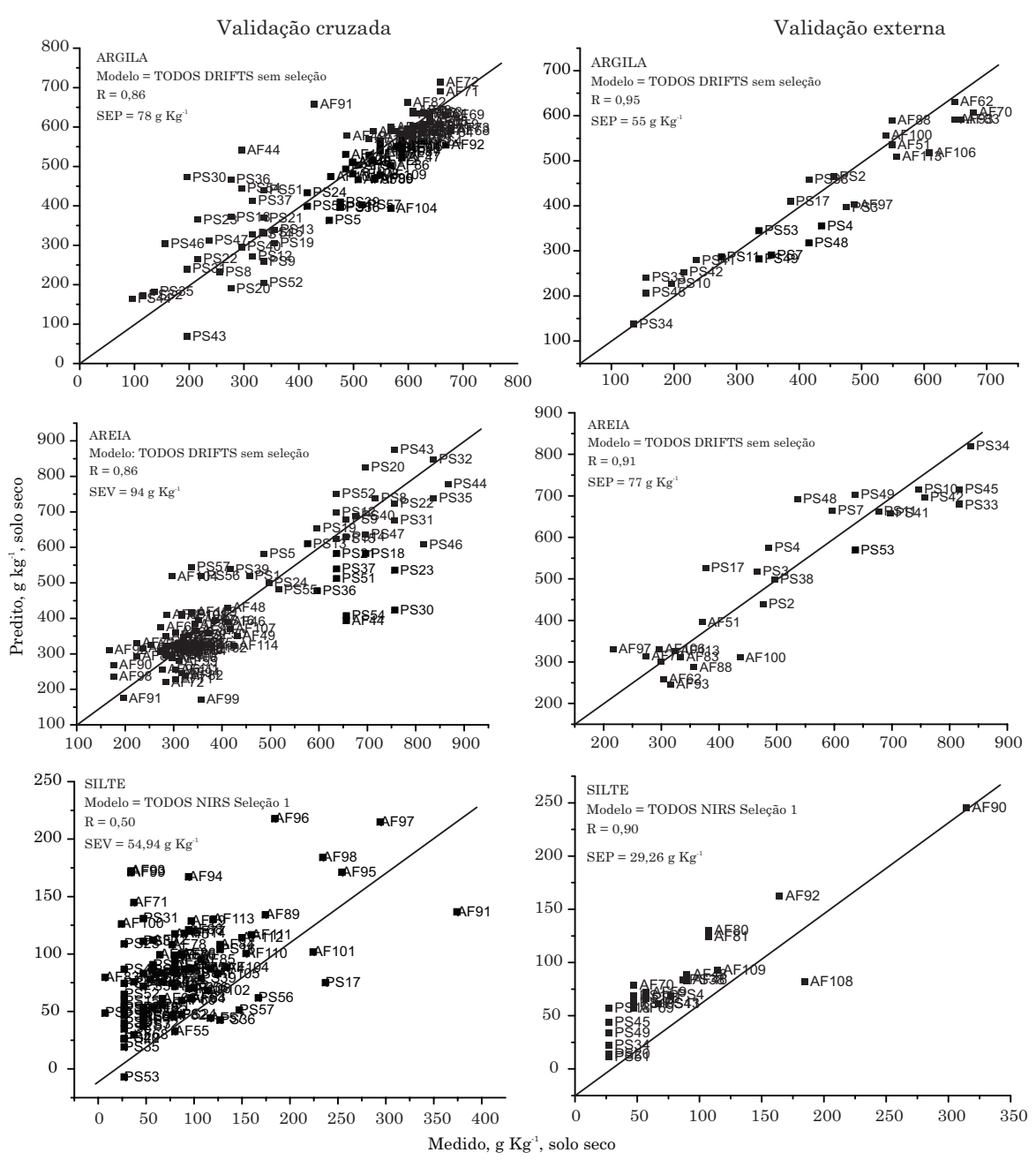

Figura 3. Valores preditos pela espectroscopia de infravermelho versus valores medidos pelo densímetro para argila, silte e areia. Linha diagonal indica situação ideal onde valores preditos são iguais aos medidos. 
pel os mel hores model os, tanto com ouso da val idação cruzada quanto da vali dação externa. Observa-se que as amostras seaproximam da linha 1:1, onde os valores preditos são iguais aos medidos, em toda a faixa analisada.

A boa correlação entre os dados medidos pelo método do densímetro e aqueles preditos pela espectroscopia, por meio de model os de calibração construídos com todas as amostras coletadas, de diferentes classes texturais etaxonômicas, indica que é possível construir modelos robustos para uso em análise granulométrica do solo. A validação externa comprova essa robustez, uma vez quea predição dos valores de argila, silte e areia conduz a uma dassificaçãotextural muito semel hanteà classificação obtida a partir dos resultados do densímetro, com acerto da classificação de 17 em 26 amostras, ou seja, $65 \%$ (Quadro 5).

Há de se considerar quealgumas amostras desolo situaram-se no limite entre duas classes texturais, o que contribuiu para a discordância entre os dois métodos. Como exemplo disso, podem-se destacar as amostras PS2, PS7 eAF 83.

\section{CONCLUSÕES}

1. A espectroscopia de infravermelho é aplicável na rotina de análise granulométrica do solo, dentro da precisão necessária para a classificação dos solos, tendo vantagens sobre o método tradicional, como: rapidez, preparo de amostra reduzido, possibilidade de automação e aumento do número de amostras analisadas por dia.

2. O número de amostras utilizado na construção do model o de cal ibração interfere mais na qual idade dos resultados preditos do quea sel eção deamostras e variáveis espectrais, sendo recomendável número maior deamostras para obter mel hores resultados nas

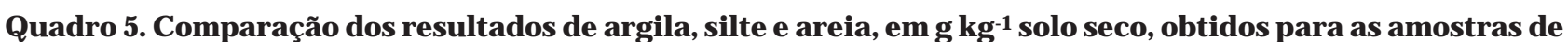
validação externa com densímetro e com a espectroscopia de infravermelho (modelo escolhido: DRIFTS com todas as amostras, sem seleção de variáveis espectrais)

\begin{tabular}{|c|c|c|c|c|c|c|c|c|}
\hline \multirow{2}{*}{ Amostra } & \multicolumn{4}{|c|}{ Densímetro } & \multicolumn{4}{|c|}{ Espectroscopia } \\
\hline & Argila & Silte & Areia & Classificação & Argila & Silte & Areia & Classificação \\
\hline PS2 & 456 & 67,2 & 476,8 & Argila arenosa & 464,8 & 86,0 & 438,8 & Argila \\
\hline PS3 & 476 & 57,2 & 466,8 & Argila arenosa & 395,9 & 72,8 & 517,7 & Argila arenosa \\
\hline PS4 & 436 & 77,2 & 486,8 & Argila arenosa & 355,1 & 51,9 & 574,6 & Argila arenosa \\
\hline PS7 & 356 & 47,2 & 596,8 & Argila arenosa & 290,5 & 32,0 & 664,9 & Franco-argilo-arenoso \\
\hline PS10 & 196 & 57,2 & 746,8 & Franco-argilo-arenoso & 226,7 & 50,9 & 715,9 & Franco-argilo-arenoso \\
\hline PS11 & 276 & 47,2 & 676,8 & Franco-argilo-arenoso & 286,5 & 42,3 & 661,7 & Franco-argilo-arenoso \\
\hline PS17 & 386 & 237,2 & 376,8 & Franco-argiloso & 410,1 & 63,9 & 526,1 & Argila arenosa \\
\hline PS33 & 156 & 27,2 & 816,8 & Franco-arenoso & 239,9 & 99,1 & 678,0 & Franco-argilo-arenoso \\
\hline PS34 & 136 & 27,2 & 836,8 & Franco-arenoso & 137,3 & 69,7 & 819,2 & Franco-arenoso \\
\hline PS38 & 416 & 87,2 & 496,8 & Argila arenosa & 458,6 & 52,6 & 497,4 & Argila arenosa \\
\hline PS41 & 236 & 67,2 & 696,8 & Franco-argilo-arenoso & 278,5 & 62,2 & 657,5 & Franco-argilo-arenoso \\
\hline PS42 & 216 & 27,2 & 756,8 & Franco-argilo-arenoso & 252,8 & 42,2 & 696,8 & Franco-argilo-arenoso \\
\hline PS45 & 156 & 27,2 & 816,8 & Franco-arenoso & 206,9 & 84,0 & 714,7 & Franco-argilo-arenoso \\
\hline PS48 & 416 & 47,2 & 536,8 & Argila arenosa & 317,5 & $-0,3$ & 690,9 & Franco-argilo-arenoso \\
\hline PS49 & 336 & 27,2 & 636,8 & Franco-argilo-arenoso & 282,2 & 10,7 & 703,4 & Franco-argilo-arenoso \\
\hline PS53 & 336 & 27,2 & 636,8 & Franco-argilo-arenoso & 344,5 & 57,2 & 569,6 & Franco-argilo-arenoso \\
\hline AF 51 & 548,8 & 80,0 & 371,2 & Argila & 534,8 & 71,3 & 395,2 & Argila \\
\hline AF 62 & 648,8 & 47,2 & 304,0 & Muito argiloso & 630,9 & 105,0 & 257,3 & Muito argiloso \\
\hline AF 70 & 678,8 & 47,2 & 274,0 & Muito argiloso & 606,3 & 76,7 & 314,4 & Muito argiloso \\
\hline AF 83 & 658,8 & 7,2 & 334,0 & Muito argiloso & 591,8 & 102,5 & 310,6 & Argila \\
\hline AF 88 & 548,8 & 94,4 & 356,8 & Argila & 589,6 & 126,4 & 287,0 & Argila \\
\hline AF93 & 648,8 & 34,4 & 316,8 & Muito argiloso & 591,7 & 163,7 & 245,7 & Argila \\
\hline AF 97 & 488,8 & 294,4 & 216,8 & Argila & 402,2 & 246,1 & 331,2 & Argila \\
\hline AF 100 & 538,8 & 24,4 & 436,8 & Argila & 556,7 & 123,1 & 310,1 & Argila \\
\hline AF 106 & 608,8 & 94,4 & 296,8 & Muito argiloso & 517,8 & 146,6 & 329,7 & Argila \\
\hline AF 113 & 556 & 120,0 & 324,0 & Argila & 508,7 & 143,5 & 326,8 & Argila \\
\hline
\end{tabular}

PS = amostra proveniente da Embrapa Pecuária Sudeste ou CPPSE. AF = amostra proveniente da Embrapa Arroz e Feijão ou CNPAF . 
calibrações, principalmente se o intuito for reunir amostras de diferentes locais com composições bastante variáveis.

3. A técnica espectroscópica mais indicada para essa análise em laboratório é a espectroscopia de infravermel ho médio (DRIFTS), tendo em vista a maior sensibilidadee precisão na predição dos teores deargila e areia no solo.

\section{AGRADE CIMENTOS}

Às instituições colaboradoras: Embrapa Instrumentação Agropecuária, Embrapa Arroz e Feijão e Embrapa Pecuária Sudeste.

\section{LITE RATURA CITADA}

CHANG, C.W.; LAIRD, D.A.; MAUSBACH, M.J. \& HURBURGH, C.R.J. Near-infrared reflectance spectroscopy-principal components regression analyses of soil properties. Soil Sci. Soc. Am. J., 65:480-490, 2001.

DALMOLIN, R.S.D.; GONÇALVES, C.N.; KLAMT, E. \& DICK, D.P. Relação entre os constituintes do solo e seu comportamento espectral. Ci. Rural, 35:481-489, 2005.

DEMATTÊ, J .A.M.; CAMPOS, R.C.; ALVES, M.C.; FIORIO, P.R. \& NANNI, M.R. Visible-NIR reflectance: a new approach on soil evaluation. Geoderma, 121:95-112, 2004.

EMPRESA BRASILEIRA DE PESQUISA AGROPECUÁRIA EMBRAPA. Serviço Nacional de Levantamento e Conservação de Solos. Manual de métodos de análise do solo. 2.ed. Rio de J aneiro, 1997. 212p.

FARMER, V.C. \& PALMIERI, F. The characterization of soil minerals by infrared spectroscopy. In: GIESEKING, J.E., ed. Soil components. New York, Springer-Verlag, 1975. p.573-670.

FORSYTHE, W. Fisica de suelos. Manual de laboratório. Costa Rica, I nstituto I nteramericano de Ciencias Agricolas, 1975. $212 p$.

GERZABEK, M.H.; ANTIL, R.S.; KOGEL-KNABNER, I.; KNICKER, H.; KIRCHMANN, H. \& HABERHAUER, G. How are soil use and management reflected by soil organic matter characteristics: A spectroscopic approach. Eur. J. Soil Sci., 57:485-494, 2006.
MADARI, B.E.; REEVES, J.B.; MACHADO, P.O.A.; GUIMARÃES, C.M.; TORRES, E. \& MCCARTY, G.W. Mid and near-infrared spectroscopic assessment of soil compositional parameters and structural indices in two Ferrasols. Geoderma, 136:1-15, 2006.

MALLEY, D.F.; MARTIN, P.D. \& DEN-DOR, E. Application in analysis of soils. In: ROBERTS, C.A.; WORKMAN J r., J . \& REEVES III, J.B., eds. Near-infrared spectroscopy in agriculture. Madison, American Society of Agronomy, 2004. p.729-784.

MINASNY, B.; McBRATNEY, A.B.; TRANTER, G. \& MURPHY, B.W. Using soil knowledge for the evaluation of midinfrared diffuse reflectance spectroscopy for predicting soil physical and mechanical properties. Eur. J. Soil Sci., 59:960-971, 2008.

PIRIE, A.; SINGH, B. \& ISLAM, K. Ultra-violet, visible, nearinfrared, and mid-infrared diffuse reflectance spectroscopic techniques to predict several soil properties. Austr. J . Soil. Res., 43:713-721, 2005.

PREVEDELLO, C.L. Física do solo: Com problemas resolvidos. Curitiba, SAEAFS, 1996. 446p.

REEVES III, J .B. Near-versus mid-infrared diffuse reflectance spectroscopy for soil analysis emphasizing carbon and laboratory versus on-site analysis: Where are we and what needs to be done? Geoderma, 158:3-14, 2010.

REIG, F.B.; GIMENO ADELANTADO, J.V.\& MOYA MORENO, M.C.M. FTIR quantitative analysis of calcium carbonate (calcite) and silica (quartz) mixtures using the constant ratio method. Appl. Geol. Samples, 58:811-821, 2002.

RESENDE, M.; CURI, N.; KER, J.C. \& REZENDE, S.B. Mineralogia de solos brasileiros - interpretações e aplicações. Lavras, Universidade Federal de Lavras, 2005. 192p.

SANTOS, G.A.; PEREIRA, A.B. \& KORNDÖRFER, G.H. Uso do sistema de análises por infravermelho próximo (NIR) para análises de matéria orgânica e fração argila em solos e teores foliares de silício e nitrogênio em cana-de-açúcar. Biosci. J., 26:100-108, 2010.

SOUSA J ÚNIOR, J .G.A.; DEMATTÊ, J.A.M. \& GENÚ, A.M. Comportamento espectral dos solos na paisagem a partir de dados coletados por sensores terrestre e orbital. R. Bras. Ci. Solo, 32:727-738, 2008.

VISCARRA-ROSSEL, R.A.; WALVOORT, T.D.J.J.; MCBRATNEY, A.B.; J ANIK, L.J . \& SKJ EMSTAD, J .O. Visible, near infrared, mid infrared or combined diffuse reflectance spectroscopy for simultaneous assessment of various soil properties. Geoderma, 131:59-75, 2006.

VUUREN, J.A.J .; MEYER, J.H. \& CLAASSENS, A.S. Potential use of near infrared reflectance monitoring in precision agriculture. Commun. Soil Sci. Plant Anal., 37:2171-2184, 2006. 